\title{
DESIGNING TOOLS FOR EXACERBATING DYSTROPHIC PHENOTYPES IN ZEBRAFISH
}

\author{
A. B. D. Thomasi*, D. F. Pires **, A. Wood*** and P. Currie*** \\ * Fundação Mineira de Educação e Cultura (Fumec), Belo Horizonte, Brazil \\ ** Pontifical Catholic University of Minas Gerais (PUC), Poços de Caldas, Brazil \\ ***Australian Regenerative Medicine Institute (ARMI), Melbourne, Australia \\ e-mail: anathomasi@hotmail.com
}

\begin{abstract}
Muscular dystrophy comprises a heterogeneous group of genetic disorders that are characterized by progressive and irreversible degeneration of muscle mass. Each of the disrupted genes distinctively affects the gene expression of one component of the two protein complexes in the muscle cell membrane: the Integrin-associated complex or Dystrophin-associated glycoprotein complex. The latter links the Extracellular Matrix to the muscle internal actin cytoskeleton, preventing fiber detachment during muscle contraction and its malfunction leads to many different diseases. One of them results from a deficiency in the glycosylation of dystroglycan, leading laminin to fail to bind. As an example, Candyfloss (LAMA2) Zebrafish has shown that fibers detach naturally as the result of basement membrane failure, therefore the hypothesis is that a similar phenomenon would be observed when exacerbated fish using a small electroshock. Fish are conventionally electrocuted using a S88 Grass Stimulator, which is not efficient, since it electrocutes only one fish at a time. For this reason, we conducted studies to develop a new device (Thomasi - Pires Lid, TPL) with a design inspired on a 48 well plate. Not only does the TPL enable the electrocution of 48 fish at a time, but it can also be used to image the fish and analyze the swimming assay on the ZebraBox, improving productivity.
\end{abstract}

Keywords: Zebrafish, Thomasi - Pires Lid, Muscular Dystrophy.

\section{Introduction}

Muscle tissue is subject to a wide range of diseases, some of which inherited, such as Muscular Dystrophies (MDs). There are more than 30 identified different genes that cause them. These diseases are characterized by the progressive degeneration of the skeletal muscle, leading to generalized muscle weakness and wasting. Unfortunately, there is no treatment to stop or revert these conditions, so the diseases remain incurable.

As a result, it is necessary to develop genetic models in an effort to understand the mechanisms involved in these illnesses. Zebrafish (Danio rerio) embryos are particularly suited to the study for many reasons: their development occurs externally, they are transparent, a large portion of their body is composed of somatic muscle, they are accessible and they begin to move at early stages of development (BASSETT; CURRIE, 2003).

Although there are many different genes to be studied, this particular article focused on the development of a new tool to exacerbate phenotypes in Zebrafish by them small shocks . Lesions in laminin subunit alpha 2 (LAMA2) cause Merosin-Deficient Congenital Muscular Dystrophy (MDC1A) (HALL et al., 2007) . The classical phenotype associated with a complete lack of LAMA2 leading to a deficiency in the glycosylation of dystroglycan in the Dystrophinassociated Glycoprotein complex (DGC). Consequently, laminin fails to bind, causing muscle fibre detachment induced by natural muscle contraction. Based on this and studies by (MANUEL et al., 2014) and (GERISCH et al., 2013), the hypothesis is that if Zebrafish are stimulated by electroshocks, muscle contraction will occur, inducing fibre detachment.

Here, we described the design of a new electric shock tool for the process. Its main objective is to increase productivity by electrocuting more fish at a time, allowing studies to achieve results faster and increasing the probability of finding a cure or treatment (drug screening) for these conditions.

\section{Materials and methods}

The development of the new technology described in this article was divided considering three main components: Design, Circuit and Program. Each of them received specific attention, so that they would work together, as it is described as follows.

Design - In order to understand our design it is necessary to know how the previous method worked. The flowchart in Figure 1, a) below shows this method.

We start the process by crossing the fish with the desired genes to be tested. When the fish are 5 days old, we electrocute them using the S88 Grass stimulator (Grass), a microcontroller and a microscope, as the fish are only $2 \mathrm{~mm}$ long (Figure 2 ).

In the next step, the fish are divided into two groups. The first group is placed in slides to be analyzed in an Abrio microscope, while the second group is taken to a 48 well plate in the ZebraBox for a swimming assay. At the end of the two procedures, all of the fish undergo a 
DNA analysis to determine and select the ones lodging the genes.

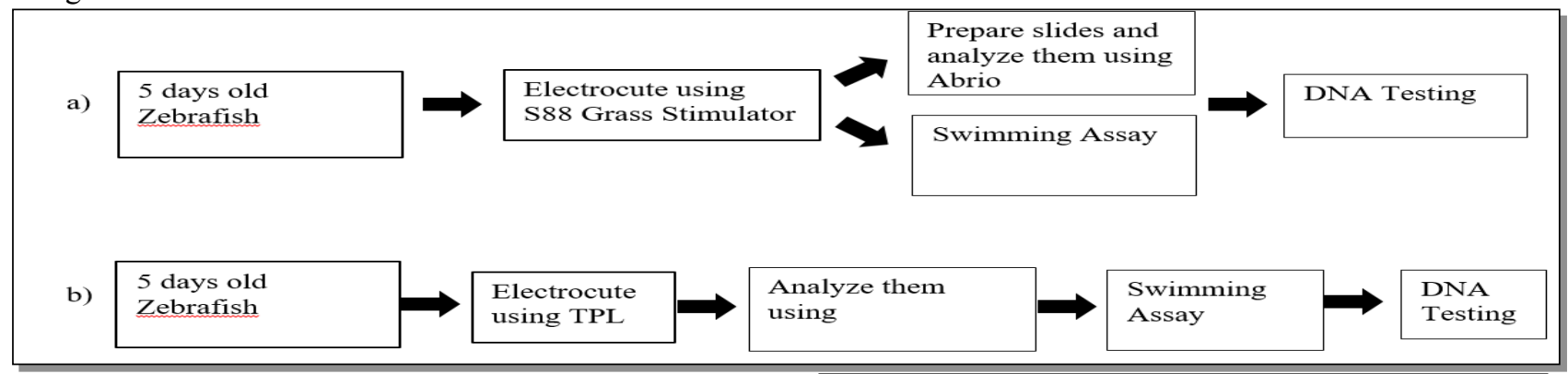

Figure 1: a) S88 Grass Stimulator process b) TPL process

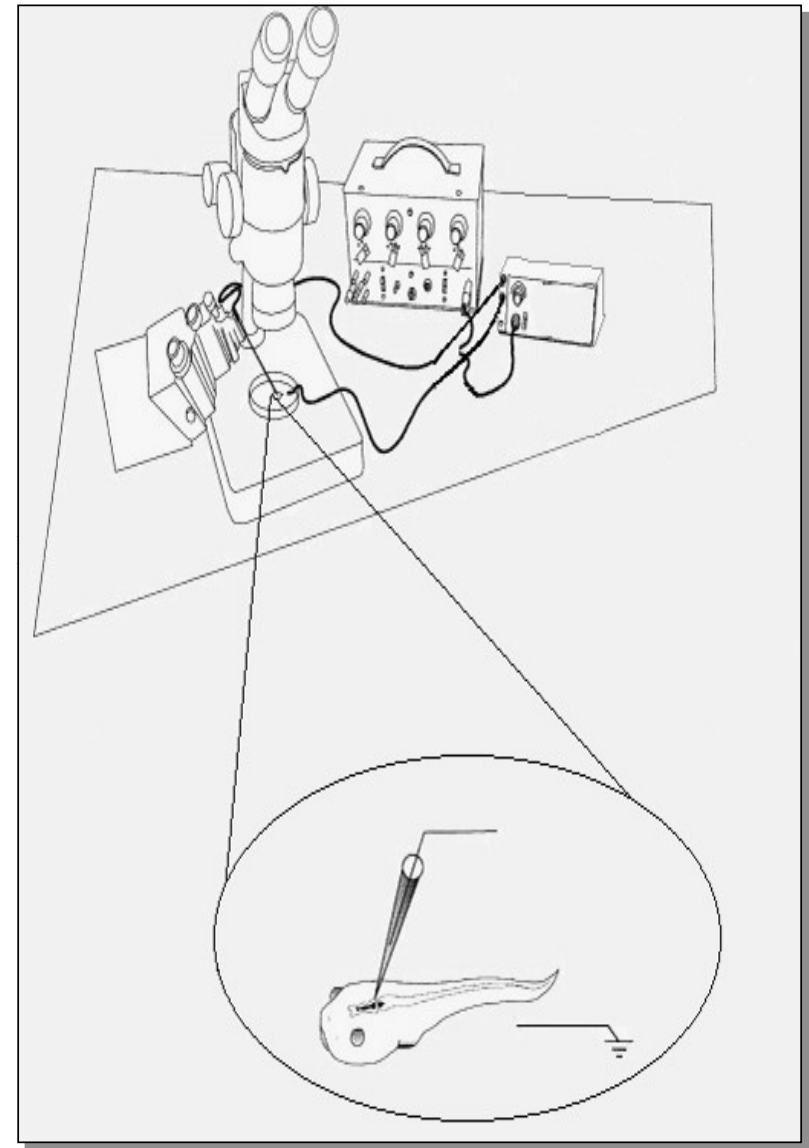

Figure 2: Electrshock using S88 Grass Stimulator

Our main concern regarding the process described is the operational limitation of Grass, as it can only electrocute one fish at a time, making the process take too long, around two days to analyze 20 fish. Furthermore, it is not possible to use the same fish in imaging and swimming assay.

Having these concerns in mind, the process described until now and represented by Figure 1a) was redesigned based on the 48 well plate (used in the last phase). Two new tools were designed using Autodesk SolidWorks to optimize the process: the Lid Separator (Figure 3) and the Lid Electroshock (Figure 4).

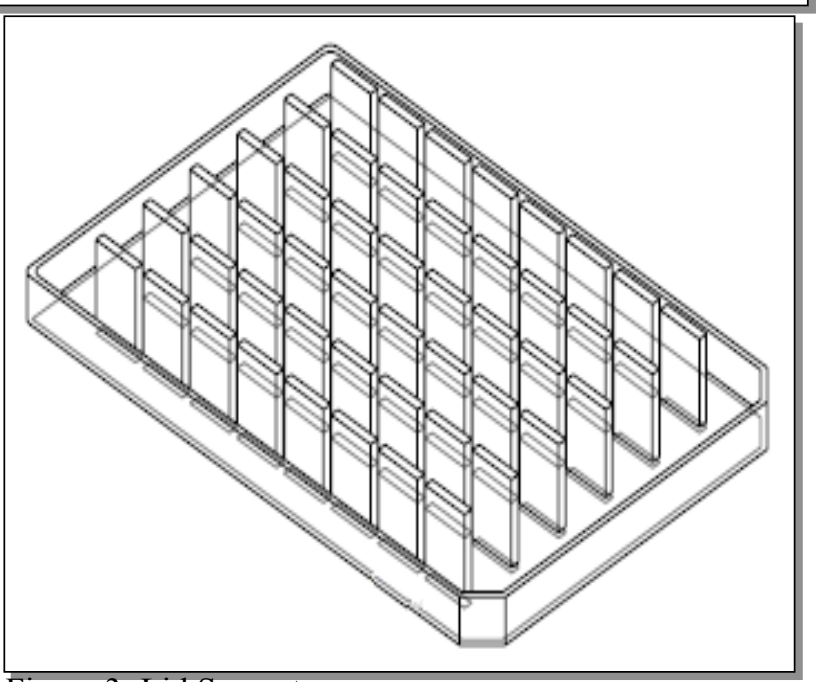

Figure 3: Lid Separator

The first one consists of a lid filled with walls placed at the top of the 48-wheel plate, reducing the space between each wheel. This measure is necessary to ensure that the fish remains still while it is being imaged in the microscope and that the shock is applied in the fish and does not disperse throughout the saline liquid. The latter is a lid filled with wholes where we will place the electrodes to apply small shocks to the fish.

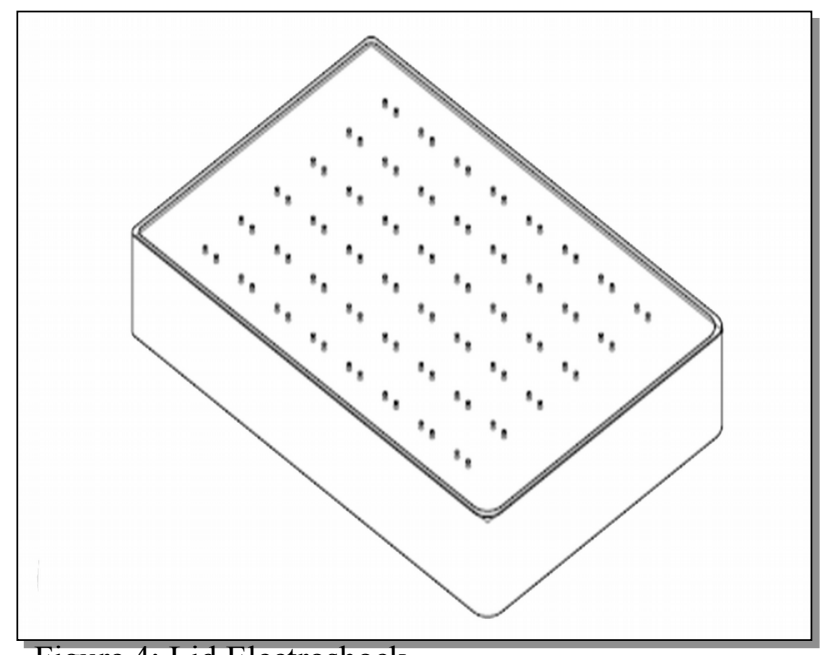

Figure 4: Lid Electroshock 


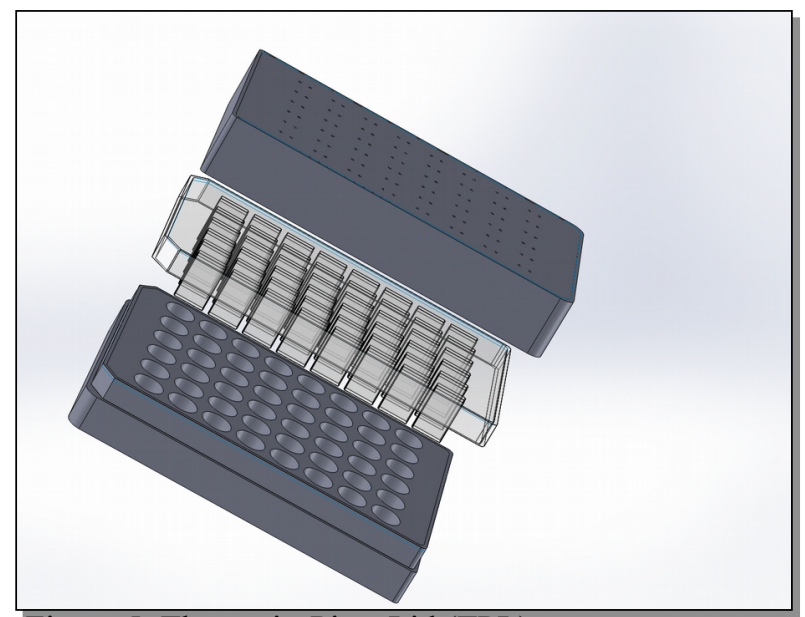

Figure 5: Thomasi - Pires Lid (TPL)

After the creation of the tools described above (Figure 5), it was possible to optimize the process illustrated by the flowchart in Figure 1a), originating a new sequence of steps shown in Figure 1b). The second process begins at the same stage as the first one, using the 5-day-old fish that are electrocuted in the next step, but this time using the new tool, Thomasi - Pires Lid.

The fish are accommodated in the middle of two walls (Lid Separator) and two electrodes, submerged in a saline solution, which enables current conduction (Figure 6). After that, the Lid Electroshock is removed and the fish are put in the Olympus to perform a microscope image processing. There is no need to prepare any slides as the Olympus microscope utilizes the 48 well plate and automatically moves around all the necessary positions. Subsequently, the Lid Separator is also removed and it more saline solution is added to the wheels, providing more space for the fish to swim in the Zebrabox. Lastly, each fish undergoes a DNA analysis to determine which ones present the desired genes.

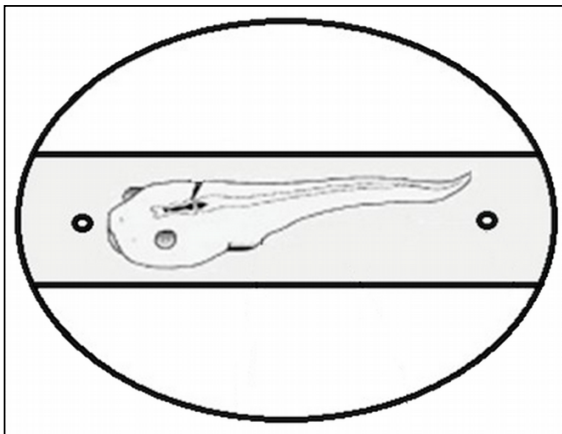

Figure 6: Accommodation of a fish in the TPL

This process is more efficient for several reasons: it electrocutes 48 fish at a time and it uses the same recipient throughout the process, improving the time spent to around 1 day.

Circuit - The first circuit designed operated in the same fashion as a dimmer, controlling the voltage delivery (Figure 7). The final circuit was improved, being controlled by an Arduino, which sets the voltage
(0 - 50V) (SUBRAMANIAN; SCHILLING, 2014), duration, repetition and interval between pulses.

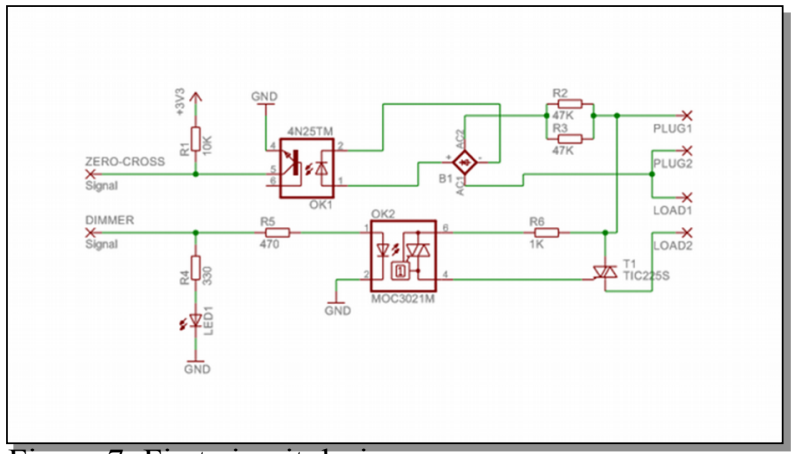

Figure 7: First circuit design

In the schematic circuit, it is important to recognize that the optocouplers are crucial components, as they have the capacity to separate the electronic circuit from the electric circuit. In other words, using them would provide a superior protection of the electronic circuit. In addition, optocouplers enhance the quality and accuracy of the measurement. We chosethe MOC3021 model, which consist of one LED and one DIAC, serving as a switch, cutting the wave like a dimmer (Figure 8).

In order to ensure that the same voltage percentage is shown in the positive and in the negative semi-circles, it must have a "Zero Crossing Detection" (Figure 9).

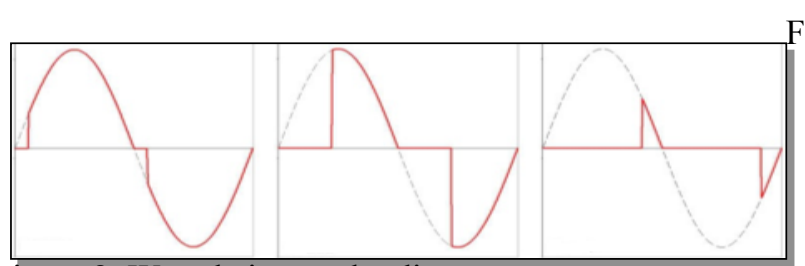

igure 8: Wave being cut by dimmer

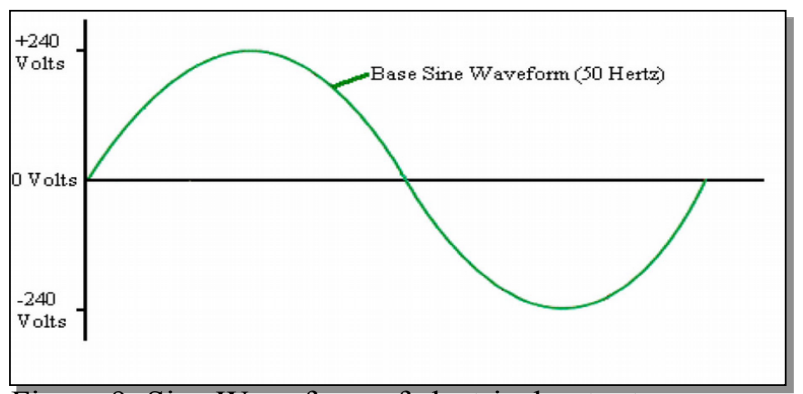

Figure 9: Sine Wave form of electrical output

This detection is made from the use of the optocoupler 4N25, which consists of one LED and one transistor. This means it can work with actuation or detection. In this case, it is used for detection. The bridge rectifier diodes rectify the wave to get a better accuracy. As a result, the LED stays on most of the time. Consequently, the transistor stays open and the zerocross signal does not receive any signal during this period. However, when the wave crosses zero, the LED turns off and sends a signal to the microcontroller. Hence, it is possible to know exactly when the wave crosses zero. 
Program - This project used the Arduino platform for programming. The following program (Figure 10) is only an example and its most important part is the function "interrupt".

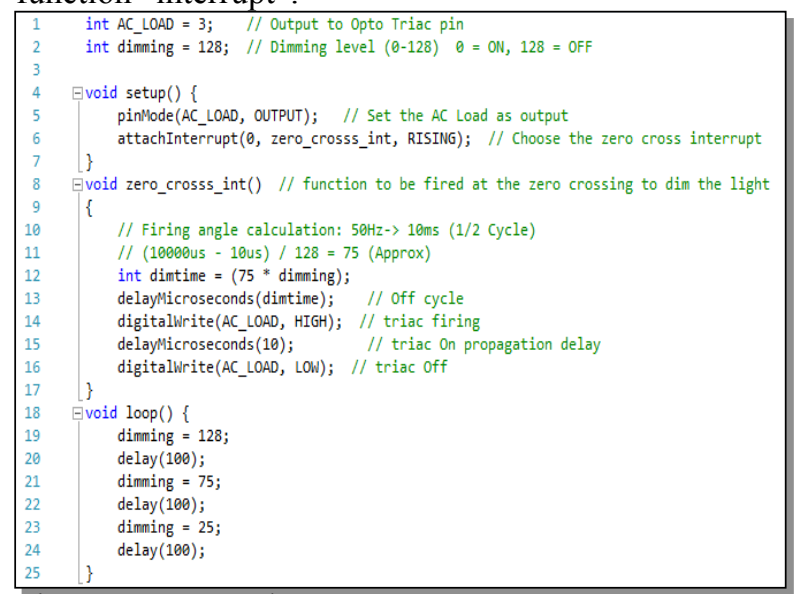

Figure 10: Example Program

\section{Results}

Figure 1clearly shows that using the TPL prototype added more efficiency to the method and accuracy to the results.

\section{Discussion}

In an effort to elucidate the mechanisms involved in genetic disorders, the main objective of this study was to develop a new electroshocking tool. It should have the ability to speed up the electroshocking step in Zebrafish.

Surprisingly, the new tool (TPL) improved not only the electroshocking step, but the entire process as it was inspired in the same plate used in both Zebrabox and Olympus microscope.

In summary, the present study suggests that the importance of this discovery is that it increases the speed of research processes related to MDs. Hence, it is plausible to expect that findings involving these disorders would be more rapidly made,raising the hopes to those who are waiting for a cure or treatment.

\section{Acknowledgements}

This piece of research was made possible through the help and support of three main institutions:

- T h e Coordenação de Aperfeiçoamento de Pessoal de Nivel Superior (Capes), which sponsored the Science Without Borders program;

- The Australian Regenerative Medicine Institute (ARMI);

- The Monash Micro Imaging.
[1] BASSETT, D. I.; CURRIE, P. D.. The zebrafish as a model for muscular dystrophy and congenital myopathy. Human Molecular Genetics, [s.1.], v. 12, n. 2, p.265-270, 22 set. 2003. Oxford University Press (OUP). http://dx.doi.org/10.1093/hmg/ddg279.

[2] HALL, T. E. et al. The zebrafish candyfloss mutant implicates extracellular matrix adhesion failure in la minin 2-deficient congenital muscular dystrophy. Proceedings Of The National Academy Of Sciences, [s.1.], v. 104, n. 17, p.7092-7097, 16 abr. 2007. Proceedings of the National Academy of Sciences. http://dx.doi.org/10.1073/pnas.0700942104.

[3] MANUEL, Remy et al. Inhibitory Avoidance Learning in Zebrafish ( Danio Rerio ): Effects of Shock Intensity and Unraveling Differences in Task Performance. Zebrafish, [s.1.], v. 11, n. 4, p.341352, ago. 2014. Mary Ann Liebert Inc. http://dx.doi.org/10.1089/zeb.2013.0970.

[4] GERISCH, G. et al. Membrane and actin reorganization in electropulse-induced cell fusion. Journal Of Cell Science, [s.1.], v. 126, n. 9, p.2069-2078, 27 fev. 2013. The Company of Biologists. http://dx.doi.org/10.1242/jcs.124073.

[5] SUBRAMANIAN, Arul; SCHILLING, Thomas F. Thrombospondin-4 controls matrix assembly during development and repair of myotendinous junctions. Elife, [s.1.], v. 3, p.1-5, 18 jun. 2014. ELife Sciences Organisation, Ltd. . http://dx.doi.org/10.7554/elife.02372.

\section{References}

